DOI: $10.5216 /$ racs.v6.65965

\title{
Investigar en equipo: fortaleciendo saberes indígenas desde la comunidad y la academia
}

\author{
Edgar Pérez Ríos ${ }^{1}$ \\ Erica Yuliana Cárdenas Vera ${ }^{2}$ \\ María Magdalena Espinosa Rivas ${ }^{3}$
}

\section{RESUMEN}

Actualmente existen diversas experiencias de investigación académica en pueblos indígenas que se sitúan desde perspectivas colaborativas. El presente artículo tiene como objetivo compartir una de esas experiencias, en el contexto de una comunidad zapoteca de la Sierra Sur de Oaxaca, México, donde realizamos nuestras tesis doctorales en educación y pedagogía. El enfoque metodológico que empleamos se sitúa desde la etnografía, en general, y desde la etnografía educativa - colaborativa en particular. Las tres tesis abordan aspectos educativos; dos de ellas en relación con procesos educativos propios de la comunidad, y otra refiere a la educación escolar. Nos interesa destacar cómo hemos articulado nuestros proyectos particulares en función de un proyecto general que tiene que ver con la construcción y fortalecimiento de saberes indígenas, en donde la propia comunidad es el agente educativo principal. En ese sentido, se trata de un ejercicio de investigación donde confluimos tanto agentes comunitarios como estudiantes de doctorado, además del acompañamiento de investigadores externos interesados en la región zapoteca de la Sierra Sur. Lo anterior invita a reflexionar en torno a la pertinencia del trabajo en equipo desde una perspectiva colaborativa, que supera la perspectiva individualista e incluso extractivista asociada a las investigaciones tradicionales.

Palabras Clave: Metodologías colaborativas. Diálogo de saberes. Pueblos indígenas.

\section{Team research: strengthening indigenous knowledge from the community and}

\section{the academy}

\begin{abstract}
Currently there are various academic research experiences in indigenous peoples that are located from collaborative perspectives. The objective of this article is to share one of those experiences, in the context of a Zapotec community in the Southern Oaxaca, Mexico, where we do our doctoral theses in education and pedagogy. The methodological approach that we use is situated from ethnography, in general, and from educational ethnography in particular. The three theses address educational aspects; two of them in relation to their own educational processes and the other related to school education. We are interested in highlighting how we have articulated our particular projects based on a general project that has to do with the construction and strengthening of indigenous knowledge, where the community itself is the main educational agent. In this sense, it is a research exercise where both community agents and doctoral students come together, in addition

\footnotetext{
${ }^{1}$ Departamento de Investigaciones Educativas del CINVESTAV, IPN, México. E-mail: edgarperezrios@gmail.com.

${ }^{2}$ Facultad de Estudios Superiores Aragón, UNAM, México. E-mail: erika_peniel@hotmail.com

${ }^{3}$ Facultad de Filosofía y Letras, UNAM, México. E-mail: magdaespinosa21@ gmail.com
} 
to the accompaniment of external researchers interested in the Zapotec region of the Southern. The aforementioned invites us to reflect on the relevance of teamwork from a collaborative perspective, which goes beyond the individualistic and even extractivist perspective associated with traditional research.

KEYWORDS: Collaborative methodologies. Dialogue of knowledge. Indigenous people.

\section{Investigar em equipe: fortalecendo o conhecimento indígena da comunidade e da academia}

\section{RESUMO}

Atualmente, existem várias experiências de pesquisa acadêmica com povos indígenas, a partir de perspectivas colaborativas. O objetivo deste artigo é compartilhar uma dessas experiências, no contexto de uma comunidade zapoteca na Serra do sul de Oaxaca, México, onde realizamos nossas teses de doutorado em educação e pedagogia. A abordagem metodológica que usamos situa-se na etnografia, em geral, e na etnografia educacional, em particular. As três teses abordam aspectos educacionais; dois deles em relação aos seus próprios processos educacionais e o outro relacionado à educação escolar. Temos interesse em destacar como articulamos nossos projetos particulares com base em um projeto geral relacionado à construção e fortalecimento do conhecimento indígena, onde a própria comunidade é o principal agente educacional. Nesse sentido, é um exercício de pesquisa em que agentes comunitários e estudantes de doutorado se reúnem, além do acompanhamento de pesquisadores externos interessados na região de Zapotec, na Serra Sur. O exposto nos convida a refletir sobre a relevância do trabalho em equipe a partir de uma perspectiva colaborativa, que vai além da perspectiva individualista e até extrativista associada à pesquisa tradicional.

PALAVRAS-CHAVE: Metodologias colavorativas. Diálogo do conhecimento. Povos indígenas.

\section{Introducción}

En México existen diversas experiencias de investigación académica con pueblos indígenas desde perspectivas colaborativas. Una de las más reconocidas en el ámbito educativo es el proyecto de Milpas Educativas, desarrollado en el estado de Chiapas mediante la articulación de educadores comunitarios e investigadores académicos, cuyo objetivo es "construir un modelo educativo intercultural multilingüe, pertinente y relevante a las prácticas productivas, laborales y ceremoniales de las comunidades indígenas" (BERTELY, 2019, p. 1). Otro proyecto colaborativo relevante es el desarrollado por maestros de una primaria bilingüe, investigadores y estudiantes de posgrado de la Universidad Nacional Autónoma de México (UNAM) y la Universidad Pedagógica Nacional (UPN) con el pueblo ikoots de San Mateo del Mar, Oaxaca, enfocado hacia la construcción de una didáctica intercultural, el cual fue coordinado y documentado por JIMÉNEZ, CARRILLO Y FRANCISCO (2019). 
Distintos autores también han reflexionado o propuesto nuevos acercamientos metodológicos en relación con la etnografía y el trabajo de campo en pueblos indígenas, con el objetivo de trascender lo que podemos denominar como etnografía tradicional. En ese sentido DIETZ (2011) habla de una etnografía colaborativa y doblemente reflexiva; CAYON (2018), desde su experiencia con los makuna en Colombia, propone una etnografía compartida; CRUZ (2018), desde el contexto brasilero, reflexiona sobre el papel de los antropólogos indígenas que realizan investigación en sus propias comunidades; por su parte, PÉREZ (2018a) ofrece una aproximación metodológica comunal en la región zapoteca de la Sierra Sur de Oaxaca. Estas propuestas y/o reflexiones propenden por un trabajo colaborativo de carácter horizontal, comprendidos también desde las metodologías decoloniales (TUHIWAI, 1999).

Bajo estas líneas, en el presente artículo nos interesa reflexionar en torno a nuestros procesos de investigación educativa, desde un enfoque colaborativo, que desarrollamos en la región zapoteca de la Sierra Sur de Oaxaca, particularmente en el Núcleo Rural "Las Palmas", perteneciente al Municipio de San Jerónimo Coatlán. Dichas investigaciones se desarrollan en el marco de nuestros estudios de doctorado; uno de ellos en el programa de Doctorado en Ciencias, con Especialidad de Investigaciones Educativas en el Departamento de Investigaciones Educativas del Centro de Investigación y de Estudios Avanzados del Instituto Politécnico Nacional (DIE - CINVESTAV), otro en el Doctorado en Pedagogía de la Facultad de Filosofía y Letras de la Universidad Nacional Autónoma de México y el último en el mismo posgrado, pero en la Facultad de Estudios Superiores, Aragón, de la misma casa de estudios.

\section{EI Núcleo Rural "Las Palmas" como contexto de investigación}

Nuestras investigaciones doctorales las realizamos en la comunidad de Las Palmas. Esta comunidad es relativamente reciente, ya que fue fundada oficialmente en 1984 mediante decreto estatal (PERIÓDICO OFICIAL, 1984, p. 43). Sin embargo, la población que conforma dicha localidad proviene de Soledad Piedra Larga, una localidad vecina fundada hacia 1930, misma que a su vez se conformó por pobladores de San Jerónimo Coatlán, un pueblo zapoteco que existe desde la época prehispánica. En ese sentido, la población de Las Palmas es, en su mayoría, originaria de San Jerónimo Coatlán, aunque actualmente nadie habla el idioma originario, el zapoteco coateco o di'zhke', ya que, de acuerdo con PÉREZ (2018b) hacia 1930, cuando se fundó 
Soledad Piedra Larga, existió un conflicto intra-étnico entre éstos y los habitantes de San Jerónimo Coatlán, cuya consecuencia fue que los de Piedra Larga decidieran explícitamente abandonar el idioma zapoteco y apropiarse del castellano, siendo ésta lengua la que se habla en la actualidad.

Siguiendo la obra de PÉREZ (2018b, 2019a), pese al cambio lingüístico ocurrido en esta población, otros elementos culturales tales como la ritualidad agrícola o los sistemas de conocimiento territoriales, tuvieron continuidad, ya que los pobladores emplearon diversas estrategias para seguir comprendiendo su entorno a partir del idioma recién adquirido. De esta forma, el territorio siguió siendo comprendido a través de la ritualidad en torno al Rayo, como máxima deidad entre los zapotecos de la Sierra Sur de Oaxaca (GONZÁLEZ, 2019). No obstante, como señala PÉREZ (2019b), en la década de 1970, tras la llegada de empresas forestales al Municipio de San Jerónimo Coatlán, la relación sagrada que los pobladores tenían con el territorio fue deteriorándose. Así, los pobladores de Soledad Piedra Larga y luego los de Las Palmas, pasaron de ser agricultores y cazadores a emplearse en el corte de madera, lo cual terminó por afectar la cosmovisión zapoteca en torno al territorio y a la naturaleza.

Actualmente en Las Palmas, como ya mencionamos, nadie habla el zapoteco coateco, aunque sí existen al menos cinco abuelitos que son recordantes, es decir, logran recordar algunas palabras, sobre todo nombres de animales, o algunas frases de cortesía. Los cinco abuelitos constituyen un bajísimo porcentaje, tomando en cuenta que la localidad cuenta con aproximadamente mil habitantes. Además del cambio lingüístico, muchas prácticas culturales, tales como los rituales agrícolas, de cacería, de nacimiento o muerte también se han abandonado. Este abandono se debe, en gran medida, al distanciamiento intergeneracional que comenzó a ocurrir a partir del cambio en las actividades laborales de la población, lo cual provocó que prácticamente se constituyeran espacios para viejos y para jóvenes (PÉREZ Y CÁRDENAS, 2020).

Como se puede advertir, el contexto actual en Las Palmas no solo tiene que ver con un cambio lingüístico, sino también con un desplazamiento en los sistemas de conocimiento tradicionales en relación con el territorio y la naturaleza. Precisamente ese es el contexto en el cual se sitúan nuestras investigaciones. Una de ellas se enfoca en el análisis de los procesos educativos tradicionales en torno al territorio; la otra aborda la transmisión intergeneracional de saberes sobre la naturaleza y la última analiza cómo se sitúan las escuelas en dicha comunidad en 
relación con los procesos socioculturales comunitarios. Las tres obedecen a un proyecto común más general, que tiene que ver con la articulación de esfuerzos dirigidos hacia el fortalecimiento o revitalización de los saberes indígenas actualmente amenazados o en riesgo de desaparición. Además, dichos proyectos han sido consensuados mediante la Asamblea de comuneros ${ }^{4}$, por tanto, obedecen también a demandas de la propia comunidad.

\section{La construcción de los objetos de estudio}

Los tres proyectos a los que aludimos en este artículo se encuentran en distintas fases. El primero, relacionado con los procesos educativos territoriales, comenzó en marzo de 2018; el segundo, que aborda la transmisión intergeneracional de conocimientos sobre la naturaleza, inició en febrero de 2019 y, finalmente, el proyecto que analiza el papel de las escuelas, comenzó en febrero de 2020. Los tres han sido planteados a partir de una problemática concreta que no tiene que ver solamente con nuestros intereses académicos, sino que dialogan con los intereses y problemáticas manifestadas desde la propia comunidad, las cuales se relacionan con el desplazamiento de una serie de saberes tradicionales sobre el territorio, la naturaleza, el bosque, el idioma originario, etcétera. De esta manera, se trata de objetos de estudio planteados en diálogo con la comunidad aquí mencionada.

Como decíamos, la forma en cómo se articulan estos proyectos fue mediante Asamblea de comuneros, en donde cada uno de nosotros expuso el tema de investigación que trabajaría, mismo que se sometió no solo a aprobación sino también a re-construcción, es decir, aunque obviamente cada uno de nosotros tiene una línea y tema de investigación, en Asamblea, nuestros temas se reconfiguraron de modo que atendieran a una perspectiva comunitaria a la vez que académica. Los comuneros, sobre todo los abuelitos, hicieron énfasis en la necesidad de "recuperar la lengua indígena", otros más dijeron que era necesario "recuperar la historia de la comunidad".

Cabe señalar que uno de nosotros es miembro de la comunidad de Las Palmas, lo cual es un punto importante a considerar a la hora de relacionarnos con la comunidad, ya que al pertenecer a dicha comunidad existe no solamente un compromiso por parte del investigador indígena sino también una responsabilidad e incluso una demanda por parte de la comunidad, que

$4 \mathrm{La}$ Asamblea de comuneros es la máxima autoridad comunitaria y se conforma por miembros de la comunidad mayores de 18 años de edad, quienes tienen voz y voto en las decisiones y acuerdos comunitarios. Cabe señalar que la condición de comunero puede ser derogada si dicha persona muestra una conducta inapropiada socialmente dentro de la vida comunitaria. 
espera que el investigador interno así como los externos retribuyan de alguna manera a la comunidad. Se trata entonces de un mutuo acuerdo, de un trabajo coordinado. Esta forma de organización también se aplica en otras aristas de la vida comunitaria, por tanto, la investigación también debe verse como una actividad comunitaria que implica a diversos miembros de la localidad.

\section{Agentes internos que participan en el proyecto}

Cuando hablamos de "comunidad" no nos referimos a una masa de personas con características e intereses uniformes. Por el contrario, dentro de la comunidad existen diversos agentes o sectores, cada cual con intereses distintos en relación con la temática general que aborda nuestro proyecto general. Así, hay quienes no están interesados en "cosas del pasado", como señalan. Otros asumen una postura neutral; aunque no rechazan el proyecto, tampoco se suman a él. Mientras que la mayoría apoya y se muestra dispuesto a participar. En ese sentido, aquí hablamos de agentes internos que están involucrados en el proyecto. A continuación describiremos a dichos agentes y el rol que juega dentro del proyecto general.

\subsection{Autoridades municipales}

Las autoridades municipales son los primeros agentes con los que entablamos conversación para llevar a cabo el proyecto. Esto se debe a que en la comunidad, previo a realizar cualquier tipo de proyecto, es necesario dar parte a las autoridades, quienes son nombradas a partir de los sistemas normativos internos, obedeciendo a la buena conducta que hayan mostrado durante su vida comunitaria. Ellos representan la voz de la comunidad, pero no pueden tomar decisiones obviando el acuerdo en Asamblea. En este caso, las Autoridades mostraron en todo momento su apoyo al proyecto; apoyo no solo a nivel moral, sino que también brindaron los espacios y herramientas necesarias, como veremos más adelante.

\subsection{Abuelitos y abuelitas}


Los abuelitos y abuelitas conforman un sector de mucha importancia para el proyecto, ya que ellos poseen una memoria histórica sobre la comunidad, así también algunos mantienen recuerdos sobre el zapoteco coateco. Los abuelitos son el grupo etario que aún conservan una serie de conocimientos tradicionales anclados al territorio y la naturaleza, por lo que son claves dentro de los objetivos que perseguimos. Ellos han mostrado una franca apertura hacia el proyecto general y los proyectos particulares, han participado en diversas actividades enfocadas hacia el fortalecimiento de los conocimientos tradicionales indígenas.

\subsection{Jóvenes}

Muchos jóvenes, hombres y mujeres de entre 14 y 40 años de edad, también se han sumado al proyecto con el objetivo de recuperar los saberes tradicionales indígenas que transmiten los abuelitos. Ellos son conscientes de la pérdida de estos saberes y de la importancia de recuperarlos. Su participación mediante el Taller Comunitario "Voces de Nuestros Cerros", que venimos implementando desde el 2018, ha sido de mucha importancia, pues los abuelitos consideran que corresponde a los jóvenes la labor de no dejar morir la cultura y la historia de la comunidad.

Dentro del trabajo de campo general, hemos diseñado el taller comunitario "Voces de Nuestros Cerros", con el objetivo de construir un espacio que permita el re-encuentro intergeneracional entre abuelitos y jóvenes. Este taller, aunque es coordinado por nosotros, en realidad los contenidos que se abordan son presentados por los abuelitos de la comunidad, así como por algunos sabios que se abocan a algún tipo de conocimientos particular. La temática del taller tiene que ver con los saberes indígenas tradicionales en torno al territorio, el bosque, la naturaleza y, en la medida de lo posible, sobre la lengua originaria.

Hasta ahora, estos tres grupos se encuentran relacionados directamente con el proyecto general, que nació en el 2018. Sin embargo, mediante el proyecto doctoral que tiene que ver con el papel de las escuelas, se pretende invitar e incorporar a las escuelas (primaria y secundaria), como agentes clave dentro del fortalecimiento de los saberes indígenas que estamos abordando.

Otros agentes internos que se han sumado indirectamente son algunas mujeres, madres de los jóvenes adscritos al taller. Ellas se han organizado para la preparación de alimentos regionales como tamales y atole para ofrecer en el marco del taller "Voces de Nuestros Cerros". Incluso, en un momento dado se sumó prácticamente toda la comunidad mediante donativos voluntarios para 
la elaboración de un mural comunitario dedicado al tema del maíz y la autonomía alimentaria, mismo que se elaboró en una casa de uso común (ver fotografía 1).

Fotografía 1 - Vista general del mural comunitario dedicado al maíz y la autonomía alimentaria.

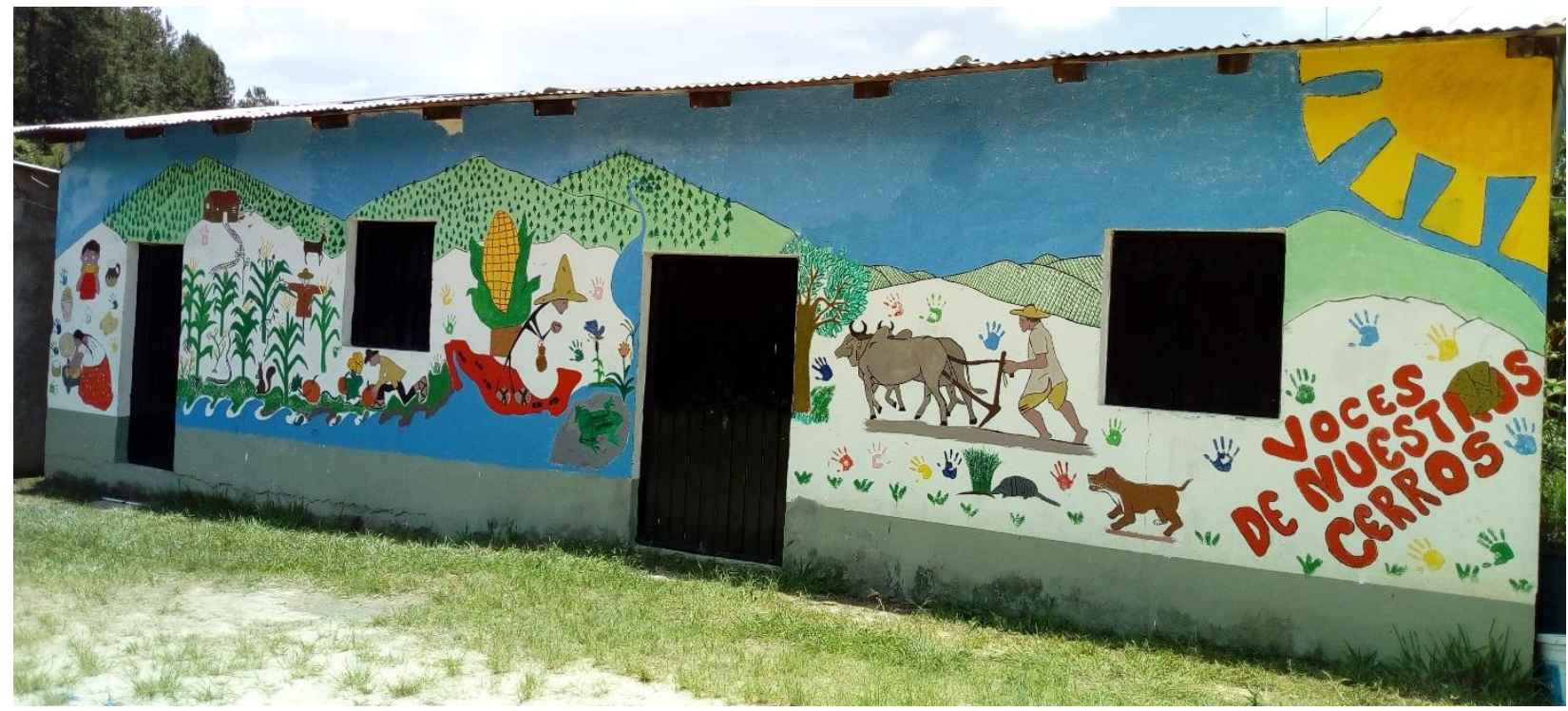

Fuente: Edgar Pérez, agosto de 2018.

\section{Agentes externos que participan en el proyecto}

Son muchos y diversos los agentes externos que participan indirectamente en el proyecto. Decimos indirectamente porque no es un proyecto "formal" o financiado por alguna institución. En ese sentido, nuestros directores de tesis y comité tutor son los principales aliados, ya que su aprobación es muy importante para la continuidad del mismo. Esto aunque pareciera ser algo lógico no lo es, pues recordemos que muchas veces los investigadores experimentados se ciñen a lógicas positivistas de hacer investigación, invalidando cualquier deseo o iniciativa de contribuir en el desarrollo de procesos educativos en donde realizamos nuestras tesis. En ese sentido también vale la pena señalar que nosotros realizamos investigación básica (no intervención) y que dicha investigación se realiza en conjunto con actores de la comunidad; de ahí que nuestros soportes metodológicos se adscriban a enfoques colaborativos, participativos, doblemente reflexivos, etcétera.

Otros agentes externos que han contribuido de distintas maneras en el desarrollo del proyecto y de manera interdisciplinar son tres especialistas: Rodolfo Rosas Salinas, desde la 
etnohistoria. Él se aboca al estudio etnohistórico de la región de Coatlán -a la que pertenece Las Palmas -durante el siglo XVI (ROSAS, 2016). Su participación ha sido de importancia para rastrear los orígenes étnicos de la comunidad, así como las reconfiguraciones socio-históricas por las que han transitado. En un par de ocasiones, Rosas ha visitado la comunidad para ofrecer conferencias en relación con su tema de estudio; dichas conferencias se han nutrido con la participación de abuelitos y adultos de la localidad.

Por su parte, la lingüista Rosemary Beam de Azcona, especialista en el estudio de la lengua zapoteca coateca o di'zhke', nos ha ayudado a identificar esta variante lingüística dentro de la familia zapotecana. Ella también ha visitado la localidad con la intención de atender aspectos relacionados con la clasificación y cambio lingüístico; también ha contribuido con la revisión de artículos sobre este tema. La obra de BEAM (2004, 2014, 2020) es una de nuestras referencias básicas para comprender los fenómenos lingüísticos en la región.

La obra del antropólogo Damián González Pérez, dedicada al análisis de la ritualidad en torno al Rayo, entre los zapotecos del sur de Oaxaca, ha sido fundamental para comprender el papel que el Rayo juega o jugaba dentro de los sistemas de conocimientos sobre el territorio y la naturaleza en la comunidad. González también ha visitado la localidad de Las Palmas y ha contribuido a comprender la naturaleza de unas piedras sagradas (gué sant) que se encuentran en los cerros aledaños, las cuales se encuentran documentadas en PÉREZ (2019a). Algunos de los datos más importantes para nuestro proyecto general se encuentran en GONZÁLEZ (2012, 2016, 2019).

Finalmente, algunos colegas y amigos se han pronunciado a favor de sumarse a este proyecto local con miras a fortalecerlo y ampliarlo a nivel municipal y de ser posible, a nivel regional, ya que la región abarca pueblos zapotecos y chatinos, los cuales comparten muchos elementos culturales en común, máxime que el zapoteco y el chatino forman parte de la misma familia lingüística otomangue. Entre estos colegas se encuentra el Doctor Antonio Carrillo Avelar de la UNAM y el Doctor Enrique Francisco Antonio, Director de la Escuela Normal Bilingüe e Intercultural de Oaxaca (ENBIO), así como los Maestros Luis Alfredo Gutiérrez Castillo, Esteban Rodríguez Bustos, Hugo Pacheco Sánchez, zapoteco de la Sierra Sur y Teresa Soriano Román, del pueblo chatino, todos ellos estudiantes del Doctorado en Pedagogía de la FES, Aragón, UNAM. Incluso a nivel internacional se ha contado con la participación de la Dra. 
Lorena Dallara Guimarães, de la Universidad Federal de Goiás (UFG), quien ha mostrado interés en sumarse al proyecto.

La importancia de trabajar a partir de un equipo más amplio radica, precisamente, en la diversidad de líneas de investigación, que pueden resultar de mucho provecho para atender otras áreas dentro de los saberes indígenas que actualmente no atendemos. Por ejemplo lo relacionado como tal con la revitalización lingüística, la implementación de estrategias didácticas interculturales o aspectos que tienen que ver con la biología y la biodiversidad local; la implementación de estrategias para el cuidado de la flora y fauna endémica de la región, etcétera. Es decir, trabajar a partir de enfoques interdisciplinarios en diálogo con otras propuestas metodológicas como podría ser el Aprendizaje Basado en Problema (ARAUJO y SASTRE, 2008), la teoría de la complejidad (MORIN, 2009) o la Pedagogía por Proyectos (JOLIBERT, 1996).

Asimismo, consideramos que nuestros trabajos en equipo, el cual toma elementos de una experiencia previa en el contexto ikoots de San Mateo del Mar, Oaxaca (JIMÉNEZ, CARRILLO y FRANCISCO, 2019), constituye una aportación metodológica a los procesos de investigación, que se vuelve colaborativo en el propio ámbito académico, en donde resulta casi inexistente. Por ejemplo, en el primer círculo de los tres investigadores que desarrollamos los proyectos de doctorado, como ya se mencionó, ha implicado un intercambio de información, de experiencias y una reflexión colectiva que enriquece los trabajos individuales y el proyecto general, y por ende la retroalimentación con la comunidad. En el segundo círculo de colaboradores externos, que con sus conocimientos, aportan insumos fundamentales que amplían el campo de observación y reflexión. Si bien en nuestras instituciones escolares de procedencia, sustentaremos la investigación de manera individual, mediante un examen de grado, esto no sería lo mismo sin dichas colaboraciones y aportaciones, hecho que quedará documentado metodológicamente en nuestras respectivas tesis.

\section{Aspectos relacionados con el trabajo de campo}

El trabajo de campo ha sido la parte más importante dentro de nuestros proyectos doctorales y, desde luego, del proyecto general. Aunque recuperamos varias recomendaciones desde la etnografía tradicional, como el diario de campo, observación y la permanencia 
prolongada en la comunidad (GEERTZ, 1973; CONKLIN, 1988; ROCKWELL, 2009), también nos abocamos a practicar un trabajo de campo desde una perspectiva colaborativa (DIETZ, 2011; CAYÓN, 2018; CRUZ, 2018; PÉREZ, 2018a). Esta forma de hacer etnografía ha redituado en que nuestras actividades dentro de la comunidad adquieren un compromiso y pertinencia social, lo cual también tiene que ver con nuestras propias conductas personales dentro de la localidad.

En primer lugar, ha sido fundamental adaptarnos a las necesidades y/o características de la comunidad y no al revés. Esto significa que dentro de la comunidad existen ciertas reglas o acuerdos tácitos de convivencia, los cuales es necesario identificar y seguir a la hora de interactuar con los pobladores. PÉREZ (2018a) llama "lenguaje comunal” a esta forma de interacción, mismas que tiene que ver, además de las conductas, con una forma de habla particular. En ese sentido, como investigadores tenemos la responsabilidad de no intentar transgredir estas formas de socialización y adaptarnos en la medida de lo posible a ellas. Recordemos que a final de cuentas las comunidades no son las que han solicitado nuestra presencia ahí, sino que hemos sido nosotros los interesados en trabajar en dicho contexto.

Siguiendo la organización comunitaria y las formas de convivencia, hemos procurado sumarnos a las actividades cotidianas; en el caso del investigador varón, realizando actividades de corte y acarreo de leña, siembra de maíz y otras actividades relacionadas con el campo. En tanto, las mujeres se han involucrado en la realización de tortillas, tamales y en otras actividades que en la comunidad son realizadas por las mujeres. También hemos participado en los tequios comunales, que son actividades de trabajo para realizar alguna obra comunitaria, como la limpieza de las calles, limpieza del río, construcción de alguna obra pública y otros ejercicios semejantes. Las investigadoras mujeres también apoyaron en la fiesta patronal a las señoras encargadas de hacer y servir la comida comunitaria que se hace en honor a la santa patrona la Candelaria.

En segundo lugar, nos ha sido importante reconocer que la comunidad tiene capacidad de agencia (ARENDT, 2003), en este caso en relación con sus procesos educativos propios. Es decir, existe desde hace siglos una educación tradicional indígena y una transmisión intergeneracional de saberes ligados al territorio y la naturaleza. Aunque, actualmente, estos han sido impactados por fenómenos externos, las propias comunidades han construido estrategias para retomar dichos procesos educativos, aunque ahora en un contexto social, económico y políticos más complejos. No obstante, consideramos que nosotros también contamos con 
herramientas, sobre todo pedagógicas y didácticas, que pueden contribuir a fortalecer los procesos de agencia en la comunidad. Es decir, es posible practicar un diálogo de saberes (LEFT, 2003) que contribuya en los procesos educativos propios, aunque, desde luego, no los suple.

Por otra parte, el trabajo de campo lo podemos dividir en dos tipos. El que cada uno de nosotros realiza en función de nuestros objetivos particulares y el que hacemos pensando en el proyecto general. En el primer caso, cada uno de nosotros trabaja con ciertos sectores de la comunidad y emplea las herramientas que mejor consideramos: entrevistas, charlas informales, etcétera; aunque, tomando en cuenta los dos puntos señalados en los párrafos anteriores. En el segundo caso, trabajos en equipo. Nos juntamos los tres para desarrollar alguna actividad conjunta. Por ejemplo, en agosto de 2019 nos reunimos con las Autoridades municipales de Las Palmas y de las demás localidades del Municipio, para acompañarlos a la demarcación de un área de reserva natural, en donde también asistieron varios jóvenes. Este evento, en particular, nos permitió observar la continuidad de la educación propia, así como las estrategias que para dicho caso emplean.

\section{El taller comunitario "Voces de Nuestros Cerros"}

En julio de 2018 comenzamos a desarrollar en Las Palmas el taller comunitario "Voces de Nuestros Cerros", de manera autogestiva, con el apoyo comprometido de las autoridades municipales, siendo en ese momento Representante Municipal es C. Ángel López Reyes y Suplente Municipal, el C. Hipólito Pérez. Se convocó a jóvenes mayores de 15 años de edad, ya que desde la perspectiva de la educación tradicional, los abuelitos consideran que a partir de esa edad los jóvenes ya están preparados para escuchar o participar de los contenidos que aborda el taller, relacionados, sobre todo, con aspectos históricos y rituales en relación con el territorio y la naturaleza. Así pues, mediante el uso de altavoces y carteles se lanzó la invitación. Tuvimos un total de 25 jóvenes inscritos, cuyas edades iban de entre los 15 y 40 años de edad. Aunque formalmente se inscribieron 25 jóvenes, en algunas de las actividades, como el caso de la elaboración del mural comunitario este número se incrementó, siendo en el cierre del taller un total de aproximadamente 100 participantes.

Uno de los temas de mayor interés para los jóvenes era respecto a la fundación de Las Palmas, por lo tanto, para dicho tema tuvimos como invitados a dos abuelos que conocen mejor 
que nadie la historia fundacional, comenzada en 1976 y concretada en 1984 mediante Decreto Estatal. En esta actividad los integrantes del taller formulamos una guía de entrevista con los temas de nuestro interés, de modo que tuvimos la oportunidad de conocer la historia en voces de sus protagonistas, pues uno de ellos, el señor Francisco Reyes, fungió como dirigente para realizar las gestiones necesarias frente al gobierno del estado, además de desempeñarse como representante municipal en los primeros años de la fundación.

Otro tema que los jóvenes consideraron relevante fue lo que tiene que ver con el di 'zhke', o zapoteco coateco. Como mencionamos, esta lengua ya no se habla en Las Palmas, por lo que nos trasladamos a la cabecera municipal, San Jerónimo Coatlán, donde aún hay algunos hablantes. Los jóvenes llevaron sus cuadernos para tomar apuntes y las abuelitas que nos recibieron estuvieron gustosas de enseñar algunas palabras en coateco. En ese mismo viaje, algunos abuelos nos relataron la historia fundacional de San Jerónimo Coatlán, pues la población de Las Palmas es originaria de ese pueblo. Los jóvenes, en general, mostraron mucho entusiasmo y disposición ante los temas planteados (ver fotografía 2).

Fotografía 2 - Los jóvenes participantes del taller platicando con abuelitos de San Jerónimo Coatlán

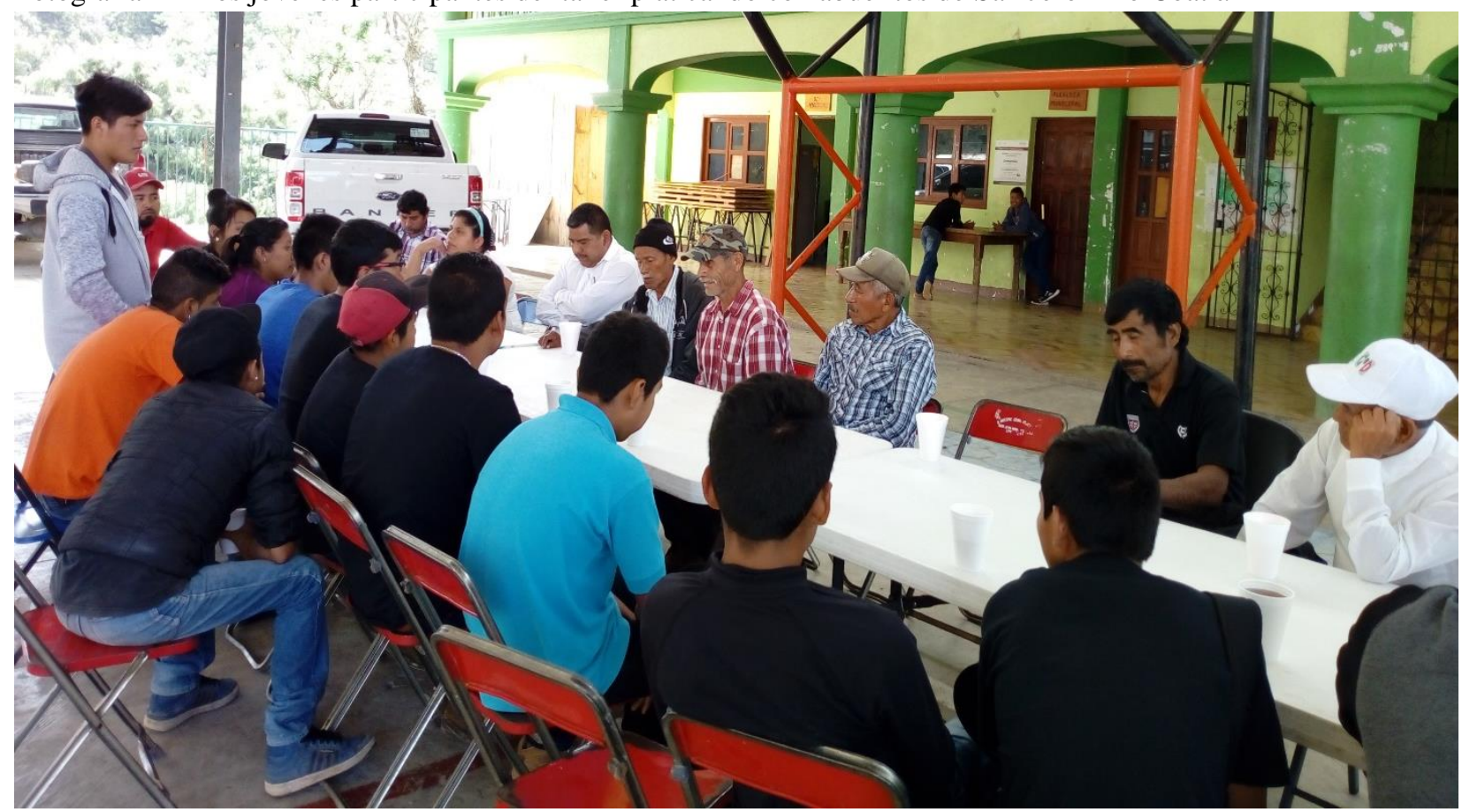

Fuente: Erica Cárdenas, julio de 2018. 
En nuestro caso estábamos aún más atentos, ya que los conocimientos que compartían los abuelitos nos servían no solo para el desarrollo de nuestros proyectos particulares, sino también para después retomarlos y analizarlos con los integrantes del taller. El uso de cuadernos de notas como el diario de campo nos permitía sistematizar la información al final de cada jornada. Así, las investigaciones avanzaban, como también avanzaba nuestra idea de investigar con relevancia y compromiso social. La idea era que entre todos los participantes construyéramos el conocimiento a la vez que lo compartíamos.

La forma de transmisión de saberes indígenas, en Las Palmas, se realiza o realizaba en espacios diferenciados a los salones de clases o auditorios, y también se expresa más allá de la lengua oral o escrita. Parte de este lenguaje se manifiesta a través de la miniatura, la cual no es exclusiva de esta comunidad, sino que se trabaja en diversas comunidades zapotecas del Sur (Elvia González, 2018, comunicación personal). El arte en miniatura es una práctica ancestral utilizada con frecuencia en rituales fúnebres, pues de acuerdo con la cosmovisión zapoteca, los muertos emprenden un camino durante nueve días hasta llegar al cielo, por lo que deben llevar algunos utensilios de uso cotidiano, así como comida, dinero y un mbèk těgăn (perro del difunto, simbolizado por una avispa metida dentro de un carrizo). Todo ello de manera simbólica, de manera que el dinero se simboliza en granos de cacao; como comida se meten nueve tortillas así como huaraches, machete y sombrero en miniatura.

Actualmente la miniatura también se utiliza en celebraciones católicas cuando se acude a realizar pedidos a la virgen o algún santo. En estos eventos las personas llevan diversas figuras de carros, caballos, casas o de aquello que los feligreses piden devotamente a sus santos. De ahí que en la comunidad la fabricación de diversos objetos en miniatura es una práctica recurrente entre niños, jóvenes o adultos. Por eso tomar en cuenta esta forma de compartir saberes responde a una estrategia didáctica comunitaria. (Ver fotografía 3).

Otra de las actividades relevantes dentro del taller fue el abordaje de saberes indígenas en relación con las gué sant (piedras sagradas), así como con la visita a un sitio prehispánico, que posiblemente fue alguna especie de adoratorio. Los abuelitos compartieron los saberes rituales en torno a dichas piedras, las cuales se complementaron con el rastreo documental que PÉREZ (2019a) realizó al respecto. Cabe señalar que estas piedras, también conocidas como guardamontes, fueron o son utilizadas con fines rituales agrícolas y de cacería (ver fotografía 4). 
Figura 3 - Dos integrantes del taller realizando una casa tradicional en miniatura

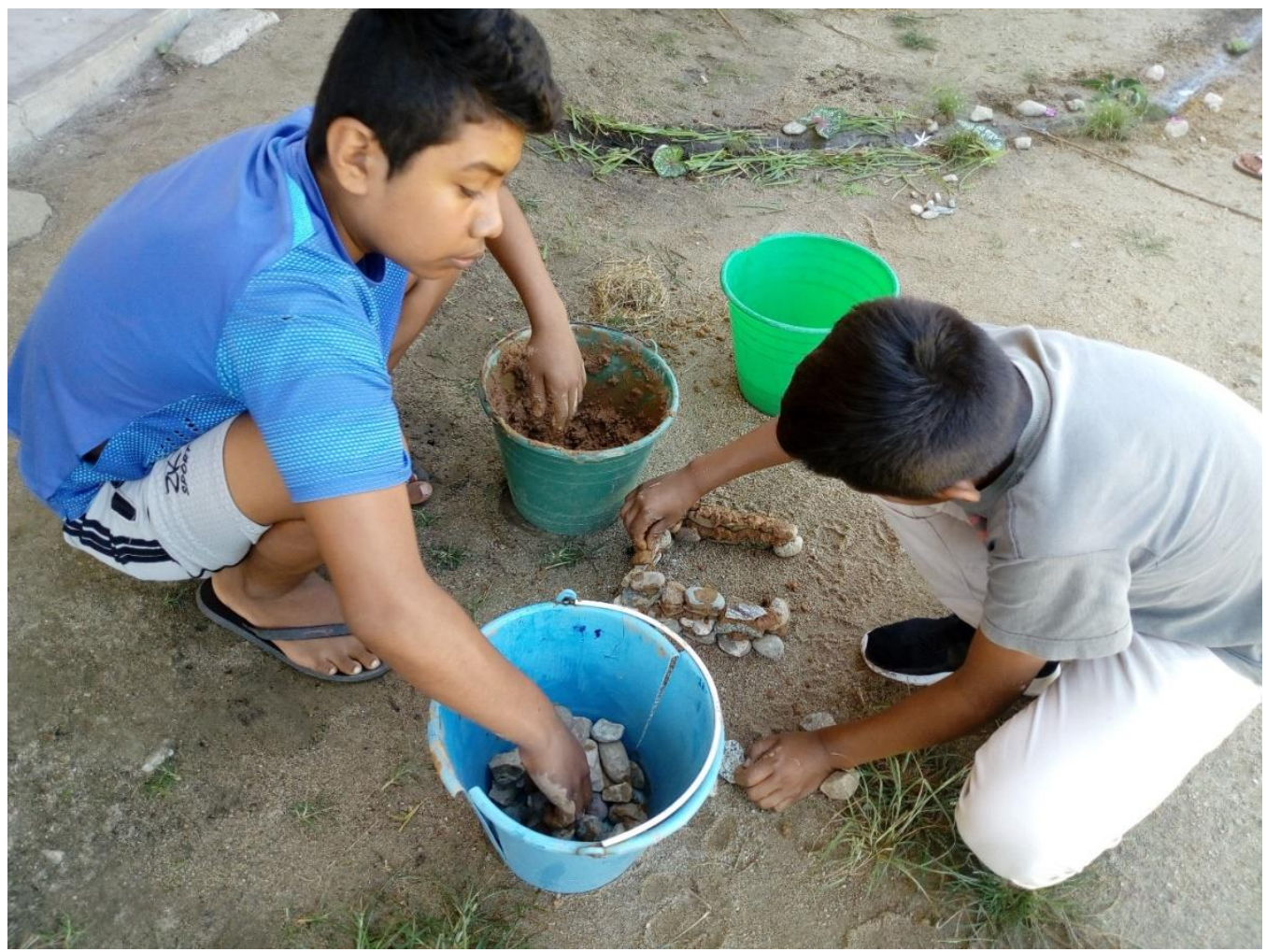

Fuente: Erica Cárdenas, julio de 2018.

Fotografía 4 - Los integrantes del taller tomando una sesión en un sitio arqueológico prehispánico

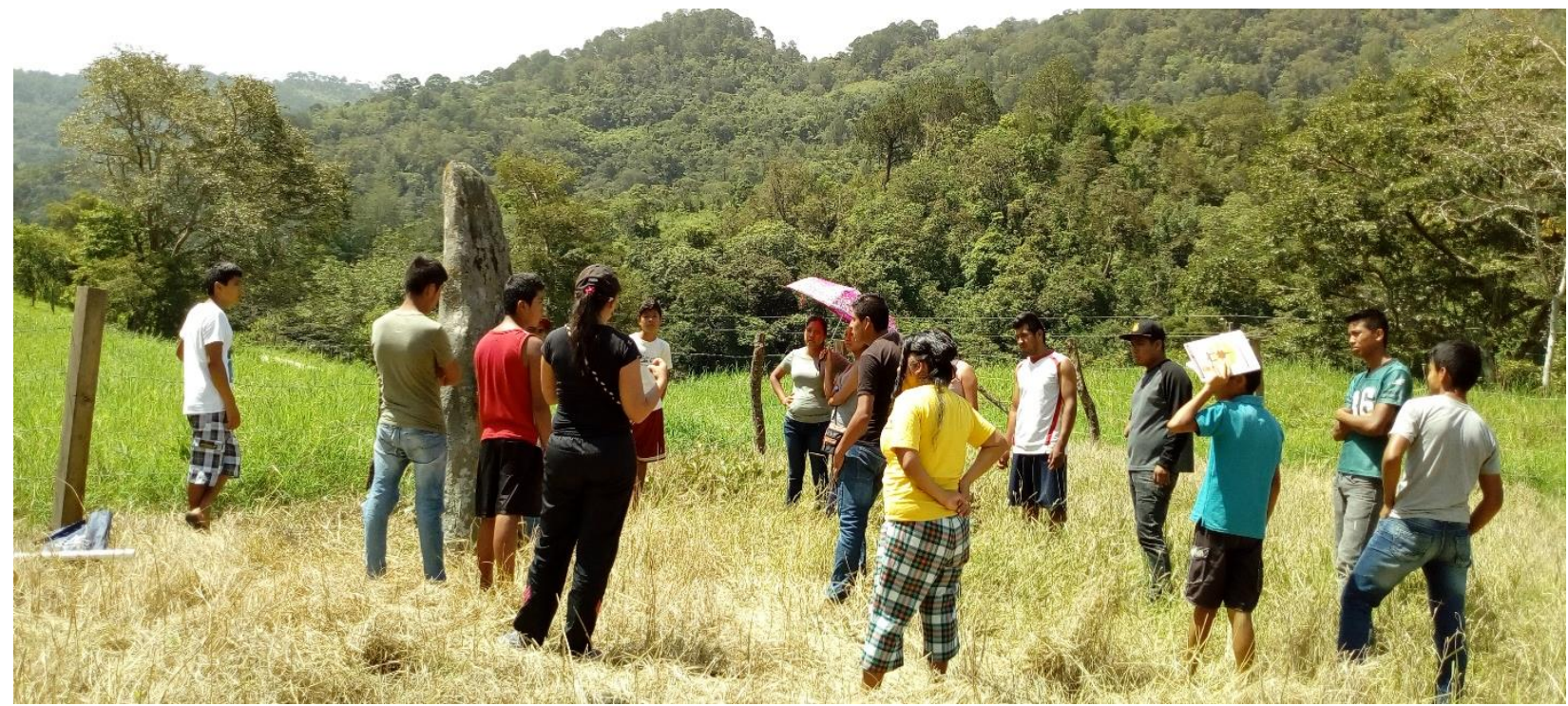

Fuente: Edgar Pérez, julio de 2018. 
En el marco del taller también tuvimos la oportunidad de participar en un ritual para la petición de las lluvias. Este ritual ha caído en desuso en Las Palmas e incluso en San Jerónimo Coatlán. Sin embargo, los abuelitos, en San Jerónimo, tuvieron una iniciativa de retomar dicho rituales, cuya última celebración fue en la década de 1970. Con apoyo de las Autoridades de Las Palmas, quienes nos facilitaron una camioneta para transportarnos, pudimos realizar el viaje a San Jerónimo Coatlán, ubicado a dos horas de camino de Las Palmas, y ser partícipes de ese momento ya histórico. También pudimos platicar con los abuelos organizadores del ritual y con el Cabildo Municipal, quienes dirigieron el evento como Principales del pueblo. Al Presidente Municipal, C. Clicerio Pérez, se le encomendó la noble tarea de colocar una luz en el nacimiento de agua, de echar humo de copal y de dirigir los rezos al Señor del Agua. Él, la Máxima Autoridad de la comunidad fue en el encargado de solicitar la lluvia (ver fotografía 5).

Fotografía 5 - Los participantes del taller (óvalo amarillo) contemplando el ritual de petición de las lluvias en San Jerónimo Coatlán.

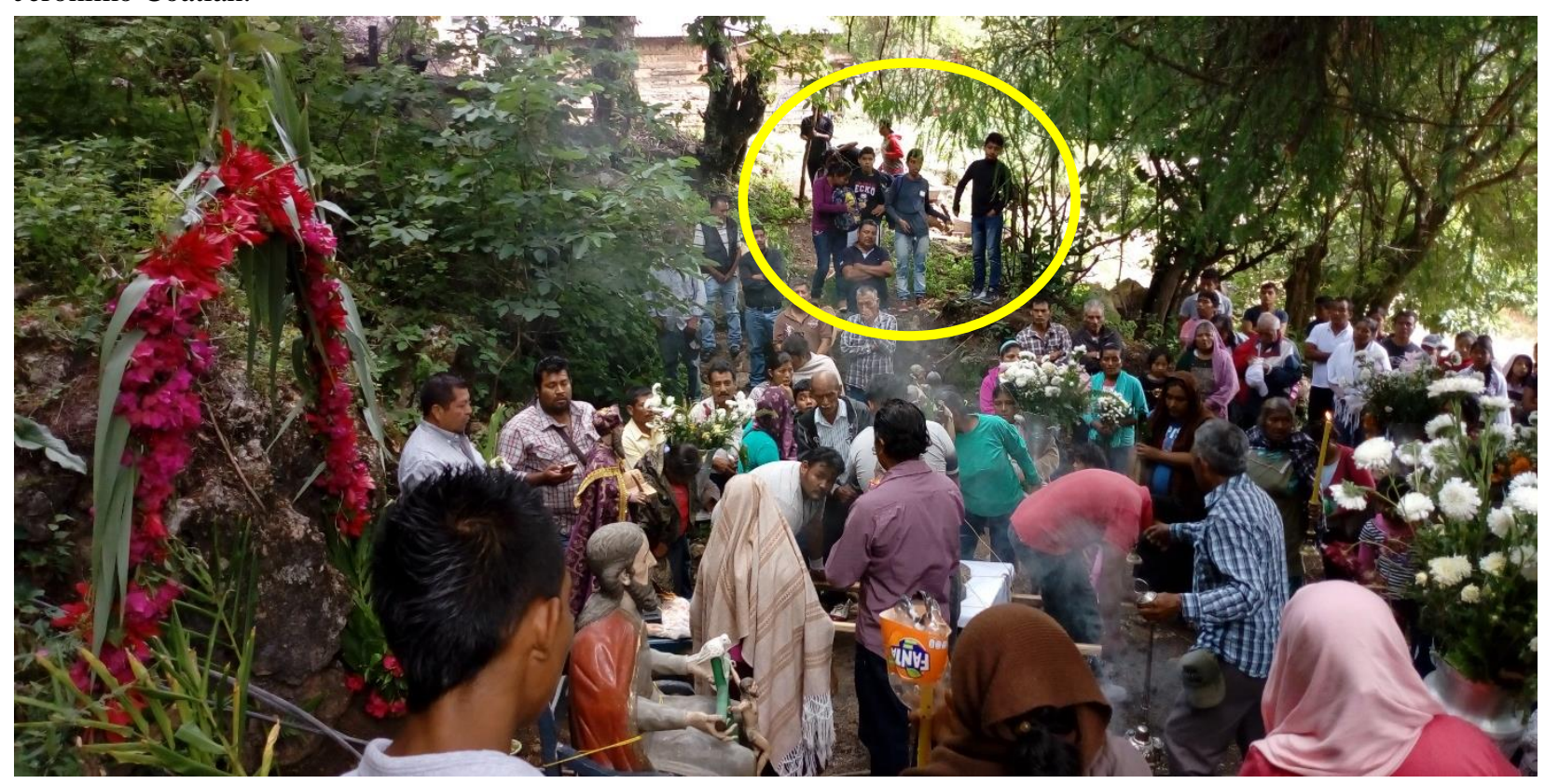

Fuente: Erica Cárdenas, julio de 2018.

Como cierre de la primera etapa del taller se propuso una actividad que reuniera la participación de toda la comunidad de Las Palmas, pintando un mural comunitario en una barda dentro del espacio que ocupa la Oficina Municipal. Esa barda guardaría una memoria de este taller y tocaría un tema emergente en la comunidad: "salvemos nuestro maíz", el cual constituye un llamado a fortalecer la autonomía alimentaria en la localidad. No teníamos pintura pero un 
integrante del taller, Juriel, propuso que se realizara una colecta en la comunidad. Se hizo la colecta y se reunieron los fondos necesarios para la pintura y las brochas (ver fotografía 6).

Fotografía 6 - Los participantes del taller elaborando el mural comunitario

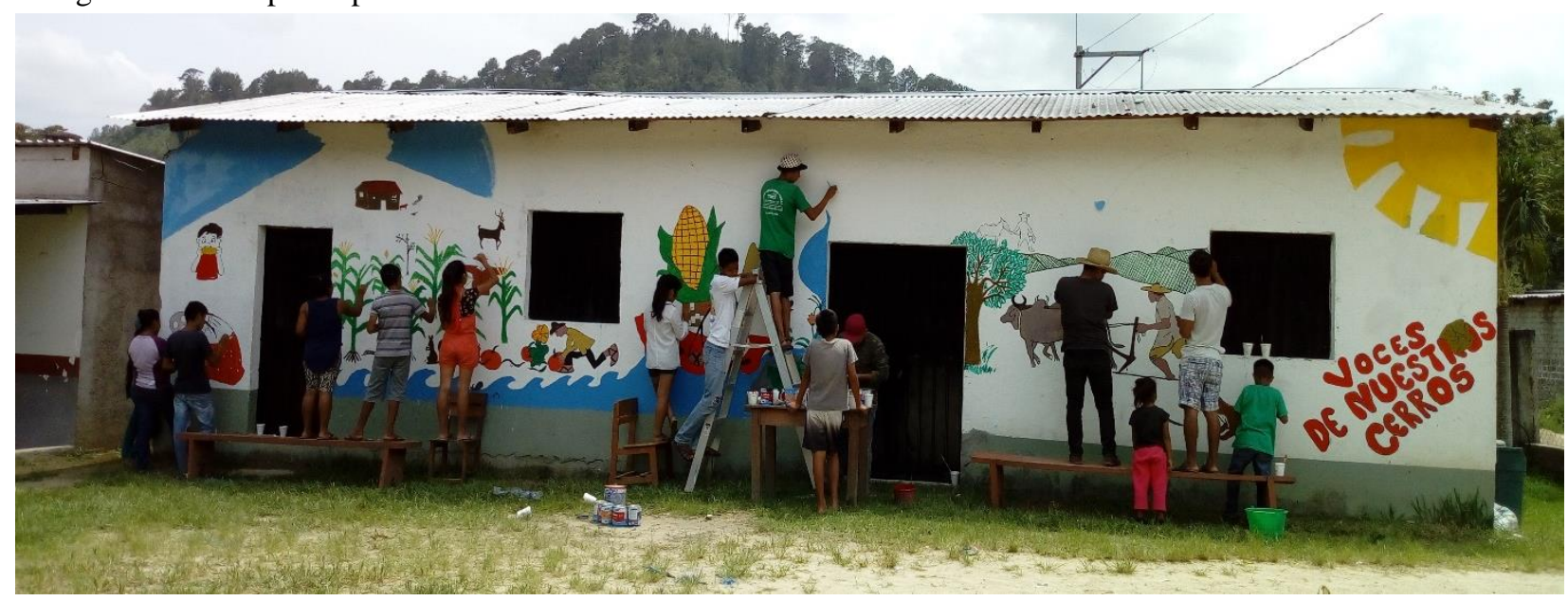

Fuente: Edgar Pérez, agosto de 2018.

Al cabo de dos días de trabajo tuvimos nuestro mural listo. Solo era cuestión de hacerle una debida inauguración a la vez que nuestra fiesta de clausura. Como toda fiesta en Las Palmas no podían faltar los tamales, así que las mamás de los participantes se encargaron de ello. Como la finalidad es practicar un diálogo de saberes entre conocimientos comunitarios y conocimientos académicos, se decidió invitar a un investigador que se ha ocupado de la etnohistoria de Coatlán, región a la que pertenece Las Palmas. De tal manera en nuestro cierre se contó con la participación de Rodolfo Rosas Salinas, quien brindó una Conferencia titulada "Historia de Coatlán", donde asistieron cerca de cien miembros de la comunidad quienes dialogaron con el conferencista y se involucraron en la discusión del tema ${ }^{5}$ (ver fotografía 7).

\subsection{Prospectiva del taller comunitario "Voces de Nuestros Cerros"}

A pesar de los esfuerzos que hemos hecho de manera personal, también hemos identificado algunos retos para fortalecer los talleres, por ejemplo, no contamos con los recursos económicos necesarios para compra de materiales y otros insumos; no contamos tampoco con el equipo adecuado para nuestras actividades, situación por la que en el 2019 no pudimos dar continuidad a las actividades, sin embargo, en este 2020 se tiene contemplado retomarlas de

\footnotetext{
${ }^{5} \mathrm{El}$ video de la conferencia completa puede verse en el siguiente enlace: https://www.youtube.com/watch?v=bqz917gEpDc\&t=4s
} 
nuevo, aunque debido a la situación sanitaria en todo el mundo, estamos a la espera de que la situación mejore. Pues incluso estamos organizado el 1er campamento internacional de coconstrucción de saberes, a celebrarse en Las Palmas tentativamente en noviembre de 2020, aunque de persistir la contingencia, se aplazaría para el año 2021.

Fotografía 7 - Asistentes al cierre del taller "Voces de Nuestros Cerros"

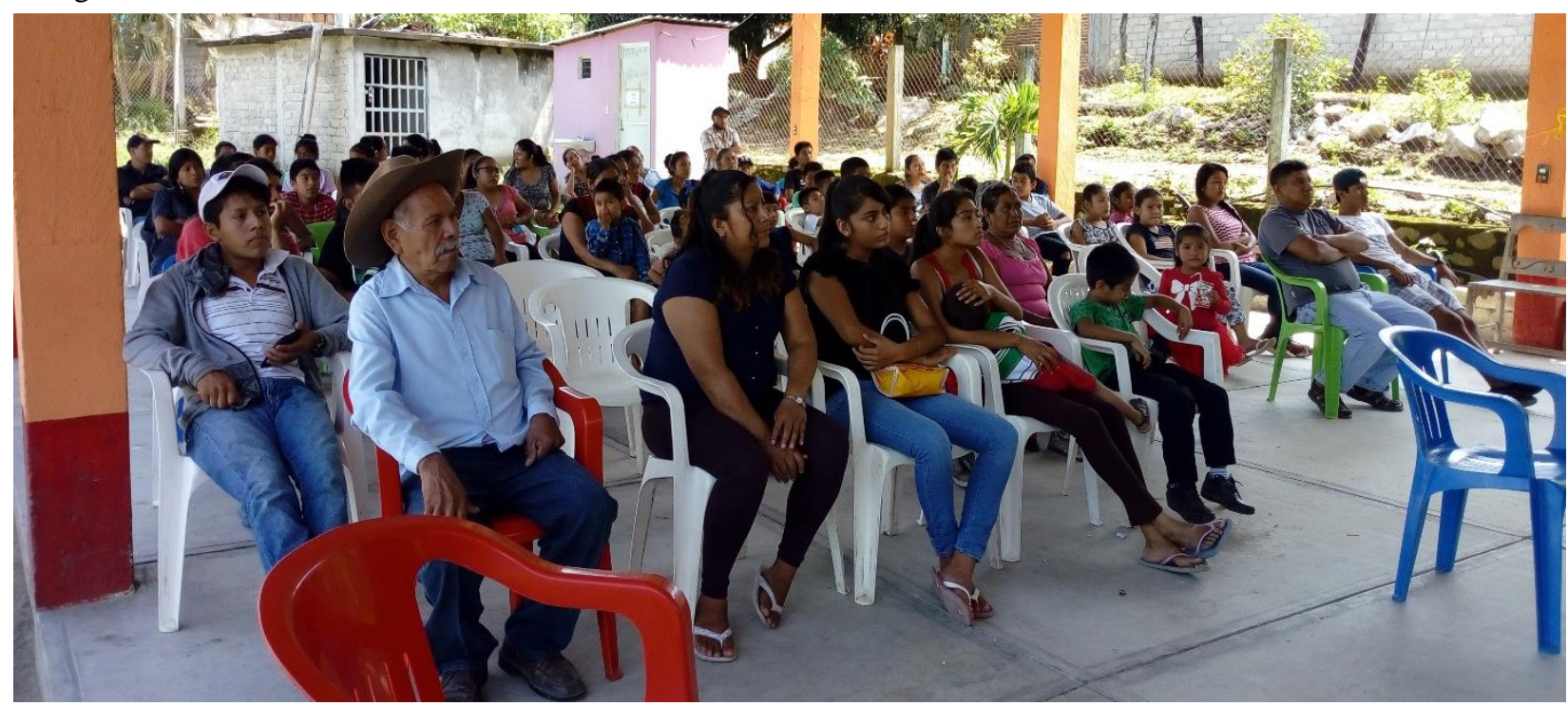

Fuente: Edgar Pérez, agosto de 2018.

\section{Una crítica a las investigaciones tradicionales - coloniales}

En contraste a esta forma de hacer investigación, hemos identificado que, por su parte, las investigaciones académicas tradicionales, enfocadas hacia las realidades socioculturales y lingüísticas de los pueblos originarios en general y particularmente en su aspectos educativos, desde perspectivas etnográficas han seguido los cánones académicos de investigación, un tanto distantes de las comunidades donde se investiga (RESTREPO 2007; TROUILLIOT, 2003).

Así pues, prevalece un trabajo académico que no ha podido dialogar con las comunidades donde se investiga. Este hecho se evidencia particularmente en trabajo de campo en comunidades originarias mediante la etnografía, que tampoco ha logrado desprenderse de los cánones académicos de "recogida de información". El etnógrafo parece contentarse con los datos que ha construido en campo, los traduce en un lenguaje académico y los comparte con sus colegas. Esto 
ha provocado la mala reputación de la investigación en los pueblos originarios, tal como señala esta intelectual maorí:

\footnotetext{
Desde el punto de vista del colonizado, posición desde la cual escribo y por la que opto, la palabra "investigación" está intrínsecamente ligado al imperialismo y colonialismo europeos. La palabra misma, "investigación", probablemente es una de las más sucias en el vocabulario del mundo indígena. En muchos contextos indígenas, cuando se menciona esta palabra, incita silencio y malos recuerdos, provoca una sonrisa que proviene del conocimiento y de la desconfianza. (TUHIWAI, 1999, p. 1).
}

Las palabras de Tuhiwai no son más que el reflejo del actual sistema colonial del saber que abarca a las instituciones académicas de producción de conocimiento científico. Son a su vez una invitación para repensar la relación academia-comunidad. Incluso diversos investigadores tanto indígenas como no indígenas desde hace unas décadas han comenzado a cuestionarse los parámetros colonizadores de la investigación académica actual, sin embargo, muchas veces estos discursan quedan en letra muerta y no termina por hacer eco en las prácticas de investigación reales.

\section{Reflexiones finales}

Quizá lo más sencillo para un estudiante de posgrado es realizar la tesis en solitario. Esto no solo es más sencillo sino también más cómodo y práctico. Nada más fácil que ir a una comunidad, entrevistar a dos o tres personas, procurar estar el menor tiempo posible y regresar cuanto antes a la ciudad, a la seguridad del escritorio, para analizar los datos en solitario, en la comodidad del hogar, quizá con una cerveza y cigarrillos al lado. Esto le evitará al estudiante lidiar con mosquitos, zancudos o tener que consumir alimentos que no son de su agrado. En realidad, nos atrevemos a pensar que esta es la perspectiva más frecuente no solo en los estudiantes sino en los mismos investigadores, llámense antropólogos, lingüistas o pedagogos. Muchos quizá piensen que el trabajo de campo es la parte sucia de la investigación.

Evidentemente nuestra postura es todo lo contrario. El trabajo de campo, la estancia prolongada en la comunidad, participar de la vida cotidiana y relacionarnos lo más profundamente posible con los pobladores es lo que da sentido a nuestros proyectos de investigación. Pensar en un trabajo en solitario incluso nos deprime. De ahí que consideramos pertinente y necesario escribir nuestras experiencias trabajando en equipo. No un trabajo en 
equipo forzado, donde se nos asigna un proyecto que debemos cumplir, sino un trabajo en equipo al cual decidimos incorporarnos por convicción, con la firme idea y anhelo de que nuestras investigaciones y formación doctoral estén permeadas del sentir comunitario.

Investigar en equipo, como intentamos mostrar aquí, es una tarea complicada. En principio por la lejanía que implica la comunidad de Las Palmas, alejada a 12 horas de viaje en autobús con respecto a la Ciudad de México, en donde se encuentran los posgrados que estudiamos. También es complicado porque se necesita reunir a las personas interesadas no solo en la región sino en la temática. Lo hace aún más complicado la falta de recursos económicos, pues nuestro proyecto general no está financiado por ninguna institución, organismo o algo parecido, por lo que todo lo costeamos con nuestros propios recursos. En ese sentido, los tres especialistas, a los que hemos invitado a la Sierra Sur, también han sido generosos en costear sus propios viajes, aun así uno de ellos, Rodolfo Rosas, nos ha acompañado en tres ocasiones, haciendo el viaje en su vehículo particular desde la Ciudad de México. Nada más gratificante para nosotros que tener aliados como él.

Otros colegas, como el Dr. Antonio Carrillo Avelar y el Maestro Luis Alfredo Gutiérrez Castillo, también han realizado el viaje a la comunidad y han manifestado su interés por contribuir en el proyecto, situación que nos anima a seguir adelante. Las propias autoridades, abuelitos y jóvenes involucrados también han expreso su deseo de continuar con las actividades en el marco de lo que hemos llamado Taller comunitario "Voces de Nuestros Cerros". Así, el equipo parece que se irá ampliando, lo cual puede redituar en un trabajo colaborativo más amplio, que abarque comunidades zapotecas y chatinas.

La idea de ampliar el equipo de trabajo es pertinente pensando en términos interdisciplinarios, puesto que la presencia de lingüistas, antropólogos, biólogos, profesores, etcétera, ayudaría a tener una perspectiva más holística sobre los procesos socioculturales y educativos en esta comunidad y región. Sin embargo, también es necesario ser cuidadosos en los objetivos que de manera particular cada uno de nosotros persigue, pues nada sería más triste que replicar prácticas de investigación coloniales que reproduzcan la idea de la comunidad como simples informantes o en el mejor de los casos como una población a quién ofrecerles algo que, desde el prejuicio, suponemos que carecen. Por el contrario, es más viable sumarnos a los procesos comunitarios ya existentes y a partir de nuestra experiencia contribuir a su fortalecimiento. 
Finalmente, hemos destacado que los saberes o conocimientos indígenas no están reñidos con los saberes o conocimientos académicos. Ambos pueden nutrirse mediante el diálogo de saberes, como hemos visto mediante el taller "Voces de Nuestros Cerros". Sin embargo, esto sigue siendo un reto en términos epistemológicos, pues aún se tiene la tarea de romper la asimetría entre los conocimientos científicos y los conocimientos comunitarios, pues mientras que éstos sirvan solo como vía para la construcción de aquéllos y no como una misma construcción, se seguirán pensando a las comunidades y sus miembros solamente como informantes o en el mejor de los casos como público a quien compartir algo. Esta asimetría deriva entonces en un no-diálogo, porque "la comunidad universitaria se asume como los "poseedores del saber' y la comunidad campesina como los 'necesitados del saber' (VÁSQUEZ, 2015, p. 150). No nos resta decir si no que sigamos haciendo equipo y sigamos fortaleciendo las lenguas y culturas indígenas de Nuestra América.

\section{Bibliografía}

ARENDT, Hannah (2003). La condición humana. Buenos Aires: Paidós.

ARAÚJO, Ulisses y SASTRE, Genoveva. (2008). El aprendizaje basado en problemas: una nueva perspectiva de la enseñanza en la universidad. Barcelona: Gedisa.

BEAM, Rosemary (2004). A Coatlan-Loxicha Zapotec grammar (Mexico). Tesis doctoral, University of California, Berkeley.

BEAM, Rosemary (2014). Algunos rasgos lingüísticos del zapoteco sureño, en Rebeca Barriga y Esther Herrera (coord.) Lenguas, Estructuras y Hablantes: Estudios en Homenaje a Thomas $C$. Smith Stark, vol. 2, México: COLMEX.

BEAM, Rosemary (2020). El zapoteco y el área lingüística de la Sierra Sur. Manuscrito inédito.

BERTELY, María (2019). Nuestro trabajo en las milpas educativas. Articulando e construindo saberes $4,1-15$.

CAYÓN, Luis (2018) Etnografía compartida: algunas reflexiones sobre el trabajo de campo con los makuna en la Amazonía colombiana. Anales de la antropología, 52, 35-43

CRUZ, Felipe (2018). Entre la academia y la aldea. Algunas reflexiones sobre la formación de antropólogos indígenas en Brasil. Anales de la antropología, 52, 25-33.

CONKLIN, Harold (1988), "Etnografía”, en Llobera, José R. (comp.) La antropología como ciencia, Buenos Aires: Anagrama, pp. 153-163.

DIETZ, Gunther (2011). Hacia una etnografía doblemente reflexiva. Una propuesta desde la antropología de la interculturalidad. Revista de antropología iberoamericana, 6 (1), 2-26. 
GEERTZ, Clifford (1973), The Interpretation of Cultures, New York: Basic Books Inc.

GONZÁLEZ, Damián (2012). "El complejo del rayo en la tradición oral de los zapotecos del sur de Oaxaca", en Malbrán, América y Enrique Méndez (coord.) Folklore y tradición oral en arqueología, vol. 1, México: Centro de Estudios Sociales y Universitarios Americanos, pp. 321343.

. (2016). De cocijo al rayo. Acercamiento etnohistórico a la ritualidad agrícola de los zapotecos del Sur de Oaxaca. Itinerarios. Revista de estudios literarios, lingüísticos, históricos y antropológicos, 24, 187-214.

. (2019). Llover en la sierra. Ritualidad y cosmovisión en torno al rayo entre los zapotecos del sur de Oaxaca, México: PUIC/UNAM.

JIMÉNEZ, María E, CARRILlO, Antonio y FRANCISCO, Enrique (coord.) (2019). Experiencias didácticas interculturales con la cultura ikoots. El caso de la Escuela Primaria Bilingüe "Moisés Sáenz" de San Mateo del Mar, Oaxaca. México: UNAM.

JOLIBERT, Josette (1996). Transformar la formación docente inicial. Propuestas en didáctica de la lengua materna. Santiago: UNESCO/Santillana.

LEFT, Enrique (2003). Racionalidad ambiental y diálogo de saberes: sentidos y senderos de un futuro sustentable. Desenvolvimento e Meio Ambiente, 7, 13-40.

MORIN, Edgar (2009). Introducción al pensamiento complejo. Barcelona: Gedisa

PÉREZ, Edgar (2018a). El lenguaje comunal como aproximación metodológica decolonial. Avá. Revista de Antropología, 33, 141-164.

. (2018b). Del dízhke' al castellano. La apropiación lingüística en una comunidad zapoteca del sur de Oaxaca. Cuadernos del Sur, 23 (45), 46-60.

. (2019a). Gué sant. Continuidad religiosa prehispánica entre los zapotecos del Sur de

Oaxaca. Itinerarios. Revista de estudios literarios, lingüísticos, históricos y antropológicos, 29, 193-213.

. (2019b). De pinos y motosierras: revisión crítica al aprovechamiento forestal comunal en San Jerónimo Coatlán, Oaxaca. Polis. Revista Latinoamericana, 52, 92-105.

PÉREZ, Edgar; CÁRDENAS, Erica (2020). De la educación rural a la educación rural comunitaria. Reflexiones desde el Municipio de San Jerónimo Coatlán, Oaxaca. Revista Latinoamericana de Estudios Educativos L (1), 225-250.

PERIÓDICO OFICIAL DE OAXACA (1984) Decreto 139 de la Quincuagésima Primera Legislatura del Estado, Tomo LXVI, Alcance al núm. 12.

RESTREPO, Eduardo (2007). "Antropología y colonialidad” En: Castro-Gómez, S. \& R. Grosfoguel. (Comps.) El giro decolonial. Reflexiones para una diversidad epistémica más allá del capitalismo global. Bogotá: Siglo del Hombre Editores; Universidad Central, Instituto de Estudios Sociales Contemporáneos y Pontificia Universidad Javeriana, Instituto Pensar, pp. 289304. 
ROCKWELL, Elsie (2009). La experiencia etnográfica. Historia y cultura en los procesos educativos. Buenos Aires: Paidós.

ROSAS, Rodolfo (2016). Quiegoqui. Un estado mene'zaa de la Sierra Sur de Oaxaca. Territorio, política y sociedad en el siglo XVI. Tesis de Licenciatura. Programa de etnohistoria, México: Escuela Nacional de Antropología e Historia.

TROUILLIOT, Michel-Ralph (2003). Transformaciones globales. La antropología y el mundo moderno, Colombia: Universidad del Cauca-Universidad de los Andes. (Traducción de Cristóbal Gnecco).

TUHIWAI, Linda (1999) Decolonizing methodologies. Research and indigenous peoples. New Zeland: University Of Otago-Zed Books Ltd.

VÁSQUEZ, Genaro (2015) Estrategia de vinculación comunitaria para la sustentabilidad: un estudio de caso en la Universidad Intercultural Maya de Quintana Roo. Tesis de maestría. Programa de investigación y desarrollo de la educación, México: Universidad Iberoamericana.

Submetido em 17 de setembro de 2020.

Aceito em 09 de fevereiro de 2021.

Publicado em 12 de março de 2021 